Artigo

\title{
Comportamento das Águas Superficiais nos Oceanos Pacifico e Atlântico Durante o Período de 1982 a 2014
}

\author{
Flaviano Fernandes Ferreira ${ }^{1,2}$, Vicente de Paulo Rodrigues da Silva ${ }^{1}$, \\ Lindenberg Lucena da Silva ${ }^{1,2}$, Emerson Mariano Silva ${ }^{3}$, José Maria Brabo Alves ${ }^{3}$, \\ Maria Marle Bandeira ${ }^{2}$, Carmen Terezinha Becker ${ }^{1,2}$ \\ ${ }^{1}$ Unidade Acadêmica de Ciências Atmosférica, Universidade Federal de Campina Grande, \\ Campina Grande, PB, Brasil. \\ ${ }^{2}$ Gerencia de Monitoramento e Hidrometria/Agência Executiva de Gestão das Águas do Estado \\ da Paraíba, Campina Grande, PB, Brasil. \\ ${ }^{3}$ Departamento de Física/Universidade Estadual do Ceará, Fortaleza, CE, Brasil.
}

Recebido: 14/1/2016 - Aceito: 14/5/2016

\begin{abstract}
Resumo
O objetivo deste estudo é analisar o comportamento das temperaturas da superfície do mar (TSM) nas áreas dos Niños no oceano Pacífico e nas áreas correspondente ao Dipolo (N-S), no oceano Atlântico, durante o período de 1982 a 2014 . Os dados utilizados foram do National Weather Service/Climate Prediction Center do National Oceanic and Atmospheric Administration (NWS/CPC/NOAA). Foi utilizado o método dos quantis para classificar os desvios normalizados da TSM acumulada de janeiro a junho como anos quentes, normais e frios. Como era de se esperar em todas as regiões dos oceanos Pacífico e Atlântico, os desvios normalizados foram positivos e acima de 1 para os anos quentes, próximo de zero para os anos normais, onde apenas a região do Niño $1+2$ apresentou um valor de $-0,28$ e valores negativos menores que -1 para os anos frios. Também foi utilizado o teste não-paramétrico de Mann Kendall para avaliar as tendências das séries temporais aos níveis de 1 e 5\% de probabilidades. E de acordo com o teste, apenas as áreas do Atlântico Norte e do Dipolo apresentaram uma tendência crescente, ao nível de significância de $1 \%$.
\end{abstract}

Palavras-chave: Niño, dipolo, TSM.

\section{Behavior of Surface Water in the Pacific and Atlantic During the Period 19822014}

\begin{abstract}
The objective of this study is to analyze the behavior of sea surface temperatures (SST) in the areas of Niños in the Pacific Ocean and in the corresponding areas to Dipole (N-S) in the Atlantic Ocean during the period 1982-2014. The data used were the National Weather Service/Climate Prediction Center do National Oceanic and Atmospheric Administration (NWS/CPC/NOAA). It used the method of quantile to classify the standard deviations of the accumulated SST January to June as hot, normal and cold years. As was to be expected in all regions of the Pacific and Atlantic Oceans, the standard deviations were positive and above 1 for the hot years, close to zero for normal years, where only the Niño $1+2$ region showed a value of -0.28 and less negative than -1 for cold years. It was also used the nonparametric Mann Kendall to assess the trends of the time series at levels 1 and 5\% probability. And according to the test, only the areas of the North Atlantic and Dipole showed an increasing trend, the level of significance of $1 \%$.
\end{abstract}

Keywords: Niño, dipole, SST.

Autor de correspondência: Flaviano Fernandes Ferreira, flavianoff@bol.com.br. 


\section{Introdução}

As anomalias da Temperatura da Superfície do Mar (TSM) representam um importante forçante dos modelos numéricos acoplados (oceano/atmosfera) que servem de ferramentas para auxiliar a previsão climática em diversas regiões do mundo. O monitoramento das condições da temperatura da superfície dos oceanos pode auxiliar na previsão climática, e assim, indicar uma possível ocorrência de seca ou chuvas intensas em uma determinada região, e com isto auxiliar nas tomadas de decisões por parte dos governantes e da sociedade organizada.

Alguns estudos mencionamque o desempenho destes modelos numéricos de previsão climática em regiões da América do Sul, como a Região Semiárida do Nordeste do Brasil (RSANEB), está diretamente relacionadoa qualidade da representação dos fenômenos associados as anomalias de TSM nos oceanos Pacifico e Atlântico Tropical, fenômeno El Niño (La Niña) e ao Dipolo do Atlântico Folland et al., 2001; Cavalcanti et al. (2002); Marengo et al. (2003); Moura e Hastenrath, 2004; Coelho, 2005; Coelho et al. (2005), (2006). Estes usam como validação os resultados de estudos observacionais que relacionaram a ocorrência de anos secos ou chuvosos na RSANEB com a ocorrência de El Niño ou La Niña no Oceano Pacífico e o sinal do Dipolo no Oceano Atlântico (Hastenrath e Heller, 1977; Moura e Shukla, 1981; Servain, 1993; Nobre e Shukla, 1996; Nobre, 2001).

Neste contexto, o presente trabalho tem como objetivo avaliar o comportamento da temperatura das águas superficiais nas últimas décadas (1982 a 2014) nos oceanos Pacífico e Atlântico. Parâmetro que, segundo a literatura, tem uma grande influência na modelagem das chuvas em diversas regiões do mundo e, em particular, na RSANEB.

Para a tarefa que se propõe foi usado o teste de Mann-Kendall para detectar a significância das tendências nas series temporais das TSM nos oceanos Pacífico e
Atlântico tropical. Um teste estatístico e não-paramétrico, apropriado para análise de tendências em séries temporais de variáveis meteorológicas e hidrológicas Yue et al. (2002); Silva, 2004; Gadgil e Dhorde, 2005; Azis, 2006 e Modarres e Silva, 2007.

\section{Dados e Metodologia}

Neste trabalho foram utilizados totais mensais de TSM dos oceanos Pacífico Equatorial e Atlântico Tropical no período de 1982 a 2014. As áreas analisadas foram Niño1+2 $\left(0-10^{\circ} \mathrm{S}\right)\left(90-80^{\circ} \mathrm{W}\right)$, Niño3 $\left(5^{\circ} \mathrm{N}-5^{\circ} \mathrm{S}\right)(150-$ $\left.90^{\circ} \mathrm{W}\right)$, Niño4 $\left(5^{\circ} \mathrm{N}-5^{\circ} \mathrm{S}\right)\left(160^{\circ} \mathrm{E}-150^{\circ} \mathrm{W}\right)$, Niño3,4 $\left(5^{\circ} \mathrm{N}-5^{\circ} \mathrm{S}\right)\left(170-120^{\circ} \mathrm{W}\right)$ (Oliveira, 1999) (Fig. 1), Índice do Atlântico Tropical Norte (TNAI), área compreendida entre $5^{\circ} \mathrm{N}-23,5^{\circ} \mathrm{N}$ e $15^{\circ} \mathrm{W}-57,5^{\circ} \mathrm{W}$, Índice do Atlântico Sul (TSAI), área compreendida entre $0-20^{\circ} \mathrm{S}$ e $10^{\circ} \mathrm{E}-30^{\circ}$ W e a área do Dipolo (TNAI-TSAI) (Fig. 2). Os dados utilizados foram do National Weather Service/Climate Prediction Center do National Oceanic and Atmospheric Administration (NWS/CPC/NOAA).

Foram calculadas as médias mensais, em seguida as anomalias, consequentemente, os Desvios Normalizados (DN) para o acumulado dos meses de janeiro a junho. Os DN foram utilizados para melhor analisarmos os valores de cada ano. O período de janeiro a junho foi escolhido para análise porque nesse período ocorrem os maiores índices de precipitação na RSANEB.

Foi utilizada a técnica dos quantis, com respeito às ordens quantificadas de $\mathrm{p}=0,25,0,50$ e $0,75(25 \%, 50 \%$ e $75 \%$ ), para classificar os DN da média acumulada da TSM de janeiro a junho, como anos quentes, normais e frios. Também foi usado o teste não-paramétrico de Mann Kendall para avaliar as tendências temporais anuais das séries temporais aos níveis de 1 e $5 \%$ de probabilidades.

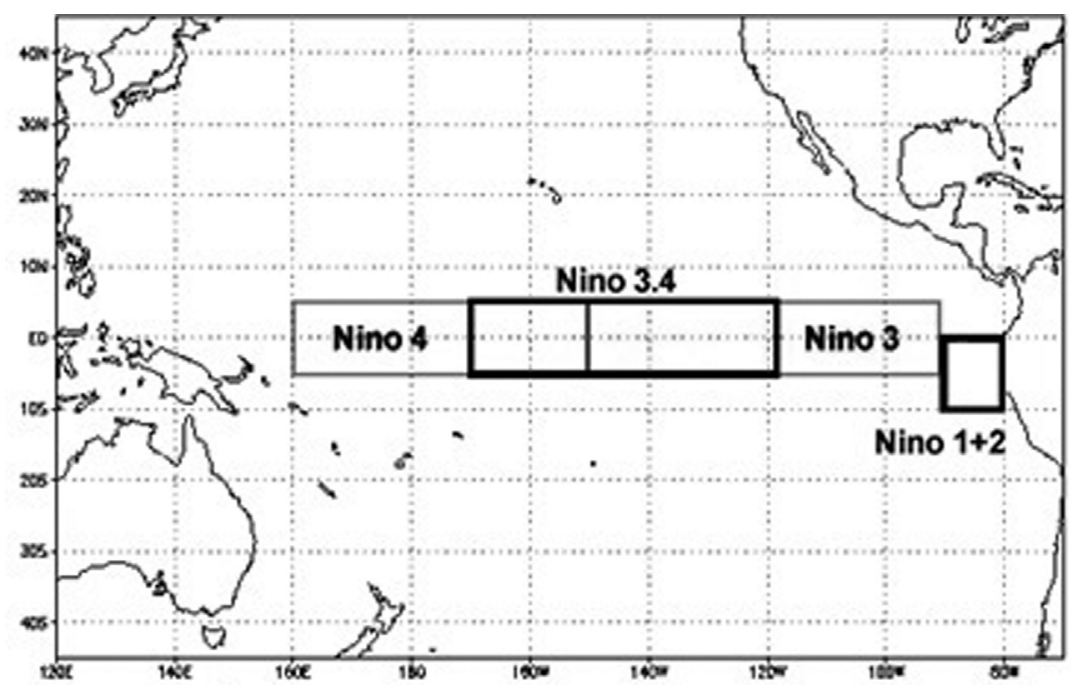

Figura 1 - Áreas do oceano Pacífico Equatorial Niño1+2, Niño3, Niño3,4 e Niño4. Fonte: Oliveira (1999). 


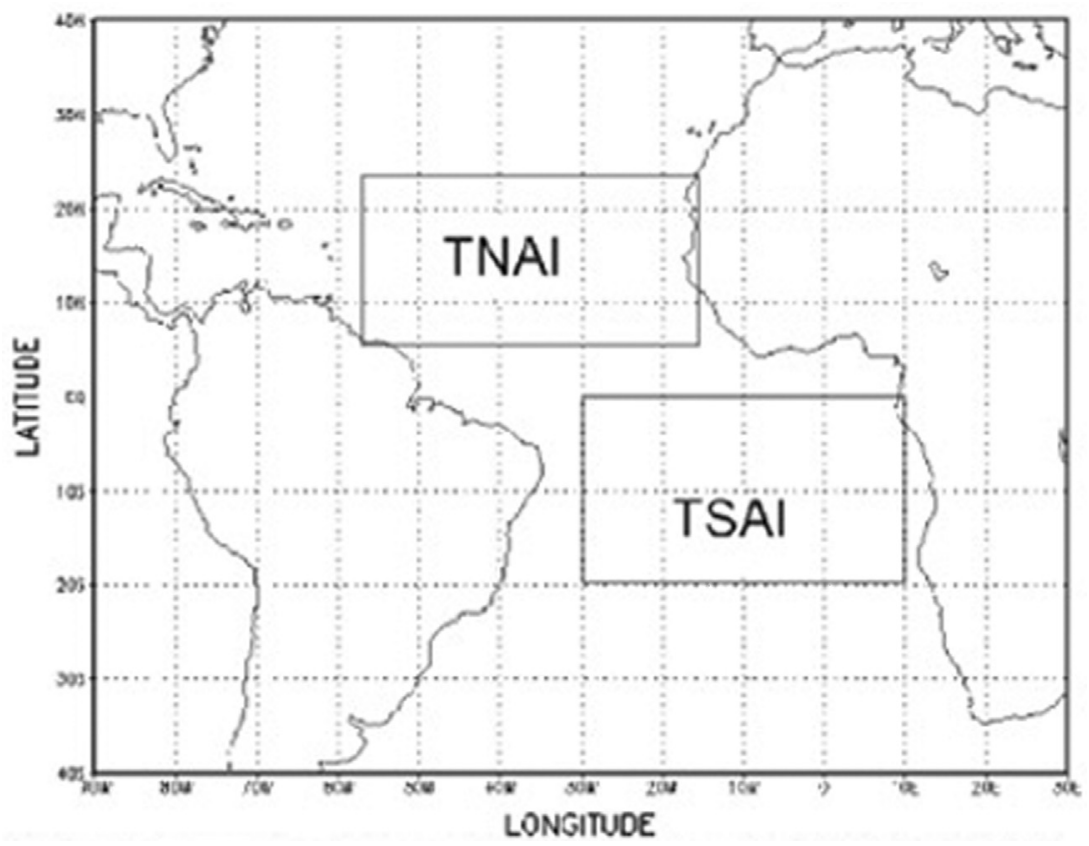

Figura 2 - Áreas do oceano Atlântico Tropical TNAI e TSAI, associados ao Padrão do Dipolo do oceano Atlântico. Fonte: (Moura e Shukla, 1981; Servain, 1991).

\subsection{Teste de Mann-Kendall}

O teste de Mann-Kendall é um teste não-paramétrico (Mann, 1945; Kendall, 1975) no qual consiste em comparar cada valor da série temporal com os valores restantes, sempre em ordem sequencial. É contado o número de vezes que os termos restantes são maiores do que o valor analisado. A estatística $S$ é obtida pela soma de todas as contagens, dado como se segue:

$$
S=\sum_{i=2}^{n} \sum_{j=1}^{i-1} \operatorname{Sinal}\left(x_{i}-x_{j}\right)
$$

em que o $\operatorname{Sinal}\left(x_{i}-x_{y}\right)$ é obtido da seguinte forma:

$$
\text { Sinal }=\left\{\begin{array}{ccc}
1 & \text { se } & \left(x_{i}-x_{y}\right)>0 \\
0 & \text { se } & \left(x_{i}-x_{y}\right)=0 \\
-1 & \text { se } & \left(x_{i}-x_{y}\right)<0
\end{array}\right.
$$

A estatística $S$ tende para a normalidade quando $n$ for grande, com média $E(S)$ e variância $\operatorname{Var}(S)$ definidas como se segue:

$$
\begin{aligned}
& E(S)=0 \\
& \operatorname{Var}(S)=\frac{n(n+1)(2 n+5)-\sum_{i=1}^{q} t_{p}\left(t_{p}-1\right)\left(2 t_{p}+5\right)}{18}
\end{aligned}
$$

em que $\left(t_{p}\right)$ é número de dados com valores iguais num certo grupo ( $p$ th) e q o número de grupos contendo valores iguais na série de dados num certo grupo $p$. O segundo termo representa um ajuste para dados censurados.

O teste estatístico parametrizado $\left(Z_{M K}\right)$ é dado por:

$$
Z_{M K}=\left\{\begin{array}{cl}
\frac{S-1}{\sqrt{\operatorname{Var}(S)}} & S>0 \\
0 & S=0 \\
\frac{S+1}{\sqrt{\operatorname{Var}(S)}} & S<0
\end{array}\right.
$$

A presença de tendência estatisticamente significativa na série temporal é avaliada usando-se o valor de $Z$. Essa estatística é usada para testar a hipótese nula que nenhuma tendência existe. $\mathrm{O}$ valor positivo de $Z_{M K}$ indica uma tendência crescente. Para testar qualquer tendência constante, crescente ou decrescente para um nível significante de $p$, é rejeitada a hipótese nula se o valor absoluto de $Z$ é maior que $Z_{1-p / 2}$, o qual é obtido na tabela da distribuição normal. Neste trabalho foram aplicados os níveis de significância de $p=0,01$ e 0,05 .

\section{Resultados}

A Tabela 1 mostra as médias acumuladas de janeiro a junho dos DN da TSM para cada ano, de acordo com o método dos quantis. Como era de se esperar em todas as regiões dos oceanos Pacífico e Atlântico, os DN foram positivos e acima de 1 para os anos quentes, próximo de zero para os anos normais, verificando que apenas a região do Niño1+2 apresentou um valor de -0,28 e valores negativos menores que -1 para os anos frios.

A Tabela 2 mostra a variação dos DN da TSM acumulada média de janeiro a junho, de 1982 a 2014 para os anos quentes, normais e frios. Ou seja, mostra os valores máximos e mínimos dos DN nas áreas estudadas. De acor- 
do com os dados, os anos mais quentes foram 1987 (Niño4 e Niño3,4), 1997 (Niño1+2 e Niño3), 2005 (Dipolo) e 2010 (áreas norte e sul do oceano Atlântico). Já os anos mais frios foram 1984 (Dipolo), 1986 (área Norte do oceano Atlântico Norte), 1988 (Niño3), 1992 (área sul do oceano Atlântico), 1999 (Niño4 e Niño3,4) e 2007 (Niño1+2).

A partir do teste não-paramétrico de Mann Kendall que serviu para avaliar as tendências anuais temporais das séries temporais aos níveis de 1 e $5 \%$ de probabilidades, nos oceanos Pacífico e Atlântico, a Fig. 3 exibe a área do Niño1+2, onde foi encontrada uma tendência linear de $-0,18^{\circ} \mathrm{C} /$ ano. Os anos de $1983\left(25,6^{\circ} \mathrm{C}\right)$ e $1997\left(25,7^{\circ} \mathrm{C}\right)$ foram os quais tiveram as maiores TSM médias anuais, ou seja, os anos mais quentes. Já o ano de $2007\left(21,9^{\circ} \mathrm{C}\right)$ foi considerado com a menor TSM média anual, ou seja, o ano mais frio.

A década mais fria foi a de 2000, com uma média de $22,9{ }^{\circ} \mathrm{C}$. já a década de 90 foi a mais quente, $\operatorname{com} 23,4{ }^{\circ} \mathrm{C}$. Apenas a tendência linear, na década de 80 , é considerada negativa, nas outras décadas e nos anos entre 2010 e 2014 , possui uma tendência linear positiva. Resalta-se que não foi aplicado o teste não-paramétrico de Mann Kendall para as tendências lineares para as décadas.

A região do Niño3, apresentou uma tendência linear de $-0,13{ }^{\circ} \mathrm{C} /$ ano, como observado na Fig. 4 que não é estatisticamente significativa ao nível de $1 \%$ pelo teste de Mann-Kendall. Os anos de 1982 e 1983 (27,0 $\left.{ }^{\circ} \mathrm{C}\right), 1987$ $\left(27,1^{\circ} \mathrm{C}\right)$ e $1997\left(27,5^{\circ} \mathrm{C}\right)$ foram os que tiveram as maiores TSM médias anuais, ou seja, os anos mais quentes. Já os

Tabela 1 - Comportamento das médias dos desvios normalizados da TSM acumulada de janeiro a junho, de acordo com o método dos quantis considerando anos quentes (são os anos quentes e muito quentes), normais e frios (são os anos frios e muito frios), nas áreas dos Niños, áreas norte e sul do Atlântico e o Dipolo.

\begin{tabular}{|c|c|c|c|c|c|c|c|}
\hline $\mathrm{DN}$ & Niño1+2 & Niño3 & Niño4 & Niño3,4 & NATL & SATL & Dipolo \\
\hline Anos quentes & 1,03 & 1,10 & 1,00 & 1,08 & 1,05 & 0,98 & 1,09 \\
\hline Anos Normais & $-0,28$ & $-0,04$ & 0,13 & 0,03 & 0,03 & 0,09 & 0,00 \\
\hline Anos Frios & $-0,87$ & $-1,03$ & $-1,21$ & $-1,13$ & $-1,09$ & 1,07 & $-1,09$ \\
\hline
\end{tabular}

Tabela 2 - Comportamento da variação dos valores máximos e mínimos dos desvios normalizados da TSM acumulada, média entre os meses de janeiro a junho, para os anos quentes e muito quentes, normais, e os anos frios e muito frios, nas áreas dos Niños, áreas norte e sul do Atlântico e o Dipolo.

\begin{tabular}{|c|c|c|c|c|c|c|c|}
\hline${ }^{\circ} \mathrm{C} /$ Ano & Niño1+2 & Niño3 & Niño4 & Niño3,4 & NATL & SATL & Dipolo \\
\hline \multirow[t]{2}{*}{ Anos quentes } & $0,34 / 2008$ & $0,51 / 1993$ & $0,65 / 2005$ & $0,58 / 1993$ & $0,33 / 1996$ & $0,44 / 1989$ & $0,41 / 1982$ \\
\hline & $2,70 / 1997$ & $2,43 / 1997$ & $1,45 / 1987$ & $2,03 / 1987$ & $2,56 / 2010$ & $1,53 / 2010$ & $2,09 / 2005$ \\
\hline \multirow[t]{2}{*}{ Anos Normais } & $-0,52 / 1984$ & $-0,51 / 2013$ & $-0,29 / 2007$ & $-0,36 / 2001$ & $-0,24 / 2001$ & $-0,19 / 2005$ & $-0,47 / 2003$ \\
\hline & $0,14 / 1991$ & $0,38 / 2006$ & $0,63 / 1993$ & $0,48 / 2003$ & $0,28 / 2003$ & $0,40 / 2011$ & $0,34 / 2006$ \\
\hline \multirow[t]{2}{*}{ Anos Frios } & $-1,34 / 2007$ & $-1,77 / 1988$ & $-2,03 / 1999$ & $-1,77 / 1999$ & $-1,65 / 1986$ & $-2,97 / 1992$ & $-2,26 / 1984$ \\
\hline & $-0,55 / 2000$ & $0,57 / 1995$ & $-0,57 / 1996$ & $-0,67 / 2010$ & $-0,43 / 2014$ & $-0,29 / 1990$ & $-0,54 / 2008$ \\
\hline
\end{tabular}

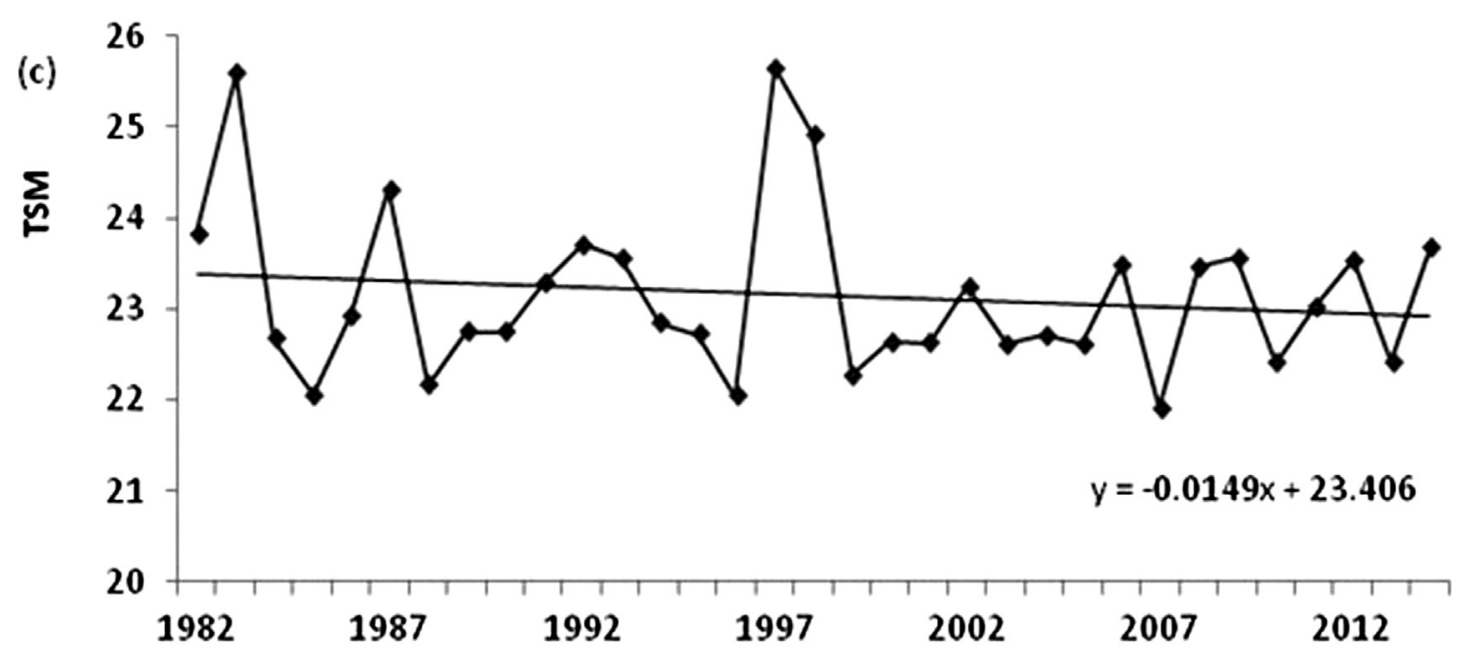

Figura 3 - Comportamento da TSM média anual na área do Niño1+2 durante os anos de 1982 a 2014, juntamente com a tendência linear. 
anos de $1988\left(24,7^{\circ} \mathrm{C}\right)$ e $1999\left(24,9^{\circ} \mathrm{C}\right)$ foram considerados com os menores valores de TSM médias anuais, ou seja, os anos mais frios. Nobrega e Santiago (2004) utilizando dados de TSM do Goddard Institute for Space Studies (GISS) compreendida entre os anos de 1950 a 2009 encontraram uma tendência positiva na área do Niño3.

A década mais fria foi a de 2000, com uma média de 25,8 ${ }^{\circ} \mathrm{C}$. já a década de 90 foi a mais quente, com uma média de $26,0^{\circ} \mathrm{C}$. As tendências lineares, nas décadas de 80 e 90, são consideradas negativas, já nas décadas 2000 e nos anos entre 2010 e 2014 possuem uma tendência linear positiva.

A região do Niño4, apresentou uma tendência linear de $-0,02{ }^{\circ} \mathrm{C} / \mathrm{ano}$, observado na Fig. 5 que não é estatisticamente significativa ao nível de $1 \%$ pelo teste de MannKendall. Os anos de $2002\left(29,3^{\circ} \mathrm{C}\right)$ e $1987\left(29,4^{\circ} \mathrm{C}\right)$ foram os quais tiveram as maiores TSM médias anuais, ou seja, os anos mais quentes. Já o ano de $1999\left(27,4{ }^{\circ} \mathrm{C}\right)$ foi consi- derado com a menor TSM média anual, ou seja, o ano mais frio.

A década mais fria foi a de 80 , com uma média de $28,4{ }^{\circ} \mathrm{C}$, enquanto a década de 90 foi a mais quente, com uma média de $28,7^{\circ} \mathrm{C}$. Apenas os anos entre 2010 e 2014 mostraram uma tendência linear positiva. Nas décadas de 80,90 e 2000, foram encontradas tendências lineares negativas.

A Fig. 6 exibe a área do Niño3,4, onde foi encontrada uma tendência linear de $-0,14{ }^{\circ} \mathrm{C} /$ ano que não é estatisticamente significativa ao nível de $1 \%$ pelo teste de MannKendall. Os anos de $1982\left(28,1^{\circ} \mathrm{C}\right), 1997\left(28,3^{\circ} \mathrm{C}\right)$ e 1987 $\left(28,4^{\circ} \mathrm{C}\right)$ foram os quais tiveram as maiores TSM médias anuais, ou seja, os anos mais quentes. Já os anos de 1988 e $1999\left(25,9^{\circ} \mathrm{C}\right)$ foram considerados com as menores TSM médias anuais, ou seja, os anos mais frios. Também os autores Nobrega e Santiago (2004) encontraram uma tendência positiva neste área do Niño3,4.

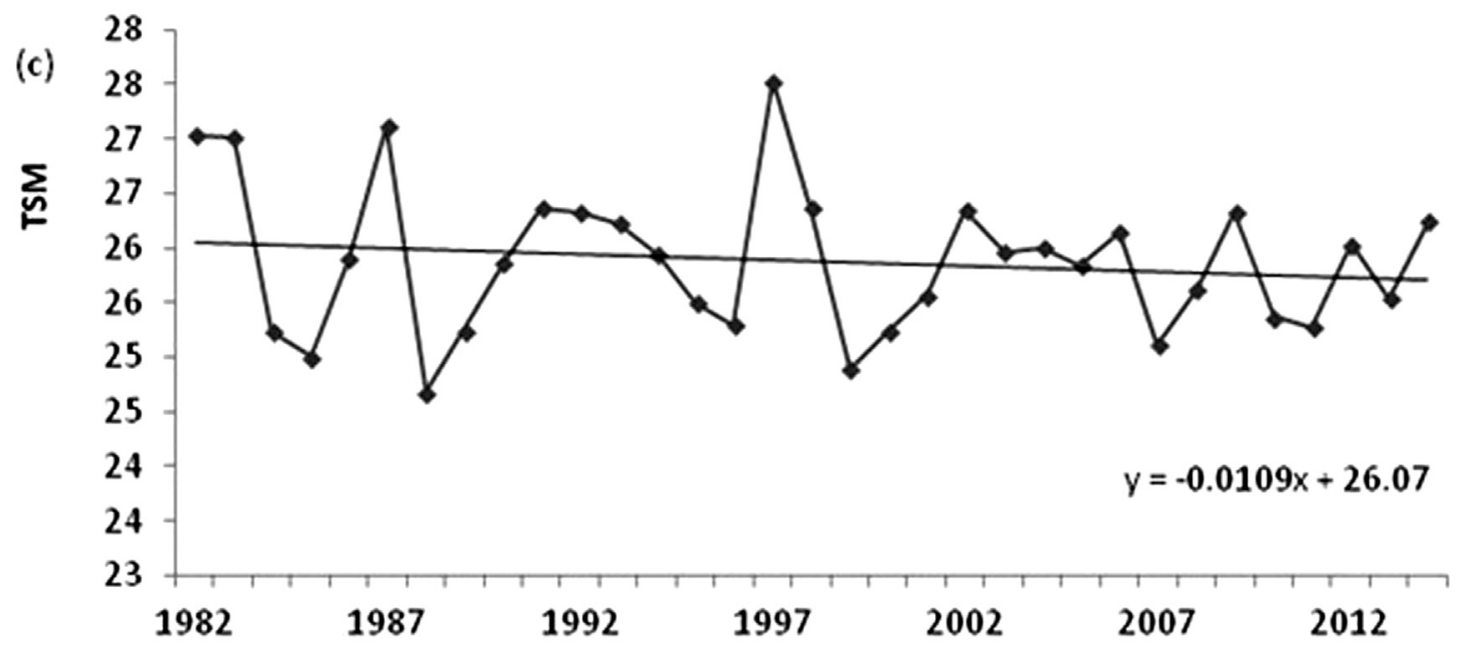

Figura 4 - Comportamento da TSM média anual na área do Niño3 durante os anos de 1982 a 2014, juntamente com a tendência linear.

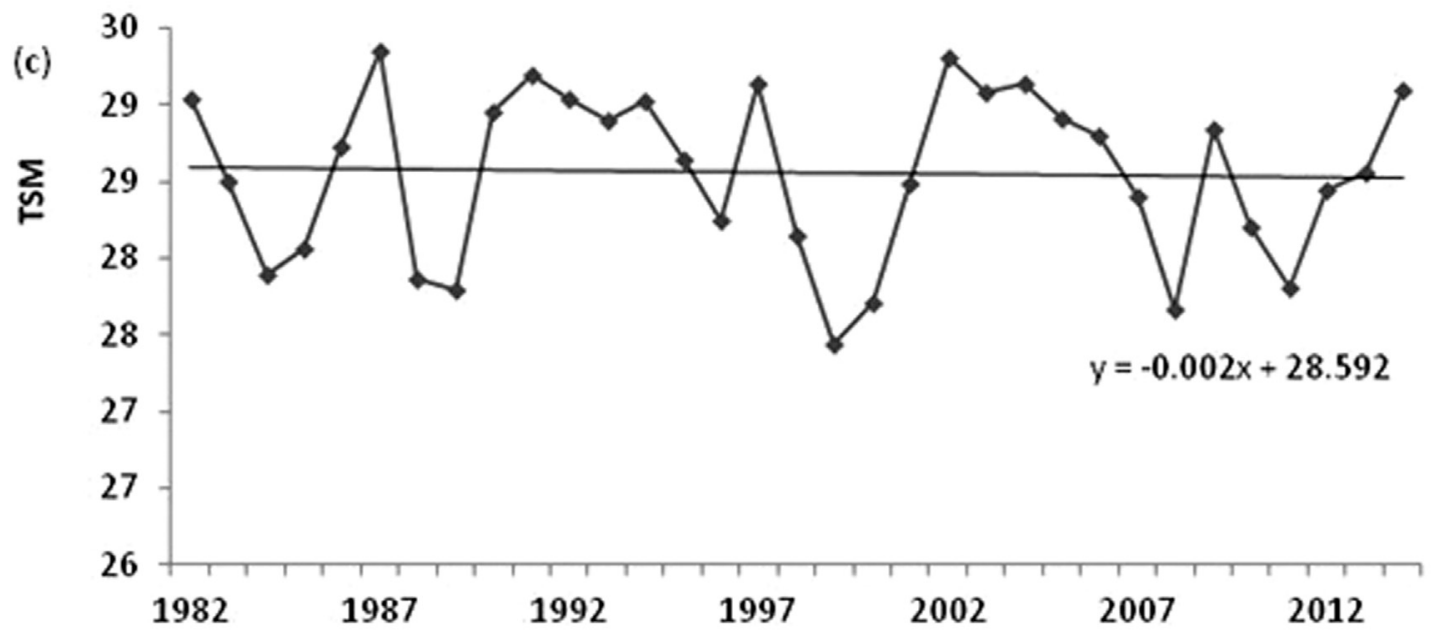

Figura 5 - Comportamento da TSM média anual na área do Niño4 durante os anos de 1982 a 2014, juntamente com a tendência linear. 
As décadas mais frias foram as de 80 e 2000 , com uma média de $27,0^{\circ} \mathrm{C}$ e já a década de 90 foi a mais quente, com uma média de $27,2^{\circ} \mathrm{C}$. A década de 2000 e os anos entre 2010 e 2014 mostraram uma tendência linear positiva, enquanto nas décadas de 80 e 90 , foram encontradas tendências lineares negativas.

A Fig. 7 exibe a área norte (Dipolo) do Atlântico, onde foi encontrada uma tendência linear de $0,27{ }^{\circ} \mathrm{C} /$ ano que é estatisticamente significativa ao nível de $1 \%$ pelo teste de Mann-Kendall. $\mathrm{O}$ ano de $2010\left(27,8^{\circ} \mathrm{C}\right)$ foi o qual se observou a maior TSM média anual, ou seja, o ano mais quente. Já os anos de 1984,1986 e $1994\left(26,3{ }^{\circ} \mathrm{C}\right)$ foram considerados com os menores valores de TSM médias anuais, ou seja, os anos mais frios.

A década mais fria foi a de 80 , com uma média de $26,6{ }^{\circ} \mathrm{C}$. Já a década de 2000 foi a mais quente, com uma média de $27,0^{\circ} \mathrm{C}$. Todas as décadas 80,90 e 2000 mostraram uma tendência linear positiva. Apenas entre os anos de 2010 a 2014, foi encontrada uma tendência linear negativa.
A Fig. 8 mostra a área sul (Dipolo) do Atlântico, onde foi encontrada uma tendência linear de $0,05^{\circ} \mathrm{C} /$ ano quenão é estatisticamente significativa ao nível de $1 \%$ pelo teste de Mann-Kendall. Os anos de 1984, 1988, 2008 e 2010 $\left(25,3{ }^{\circ} \mathrm{C}\right)$ foram os quais se observaram as maiores TSM médias anuais, ou seja, os anos mais quentes. Por outro lado, o ano de $1992\left(24,2^{\circ} \mathrm{C}\right)$ foi considerado com a menor TSM média anual, ou seja, o ano mais frio.

A década mais fria foi a de 90 , com uma média de $24,9^{\circ} \mathrm{C}$ e as décadas de 80 e 2000 foram as mais quentes, com uma média de $25,0^{\circ} \mathrm{C}$. Todas as décadas 80,90 e 2000 mostraram uma tendência linear positiva. Apenas entre os anos de 2010 a 2014, foi encontrada uma tendência linear negativa. Considerando que não foi aplicado o teste nãoparamétrico de Mann Kendall para as tendências lineares para as décadas.

Na Fig. 9 exibe observa-se a região do Dipolo (N-S) do Atlântico, onde foi encontrada uma tendência linear de $0,05^{\circ} \mathrm{C} /$ ano que é estatisticamente significativa ao nível de

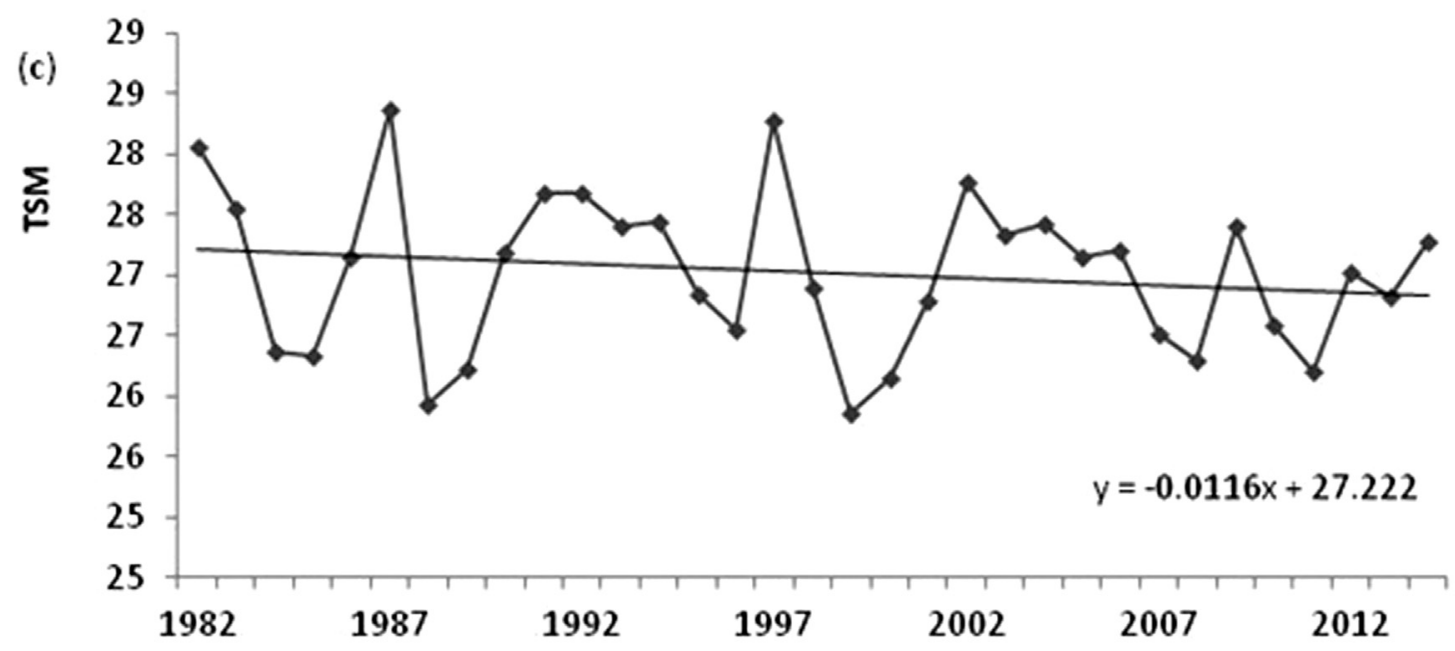

Figura 6 - Comportamento da TSM média anual na área do Niño3,4 durante os anos de 1982 a 2014, juntamente com a tendência linear.

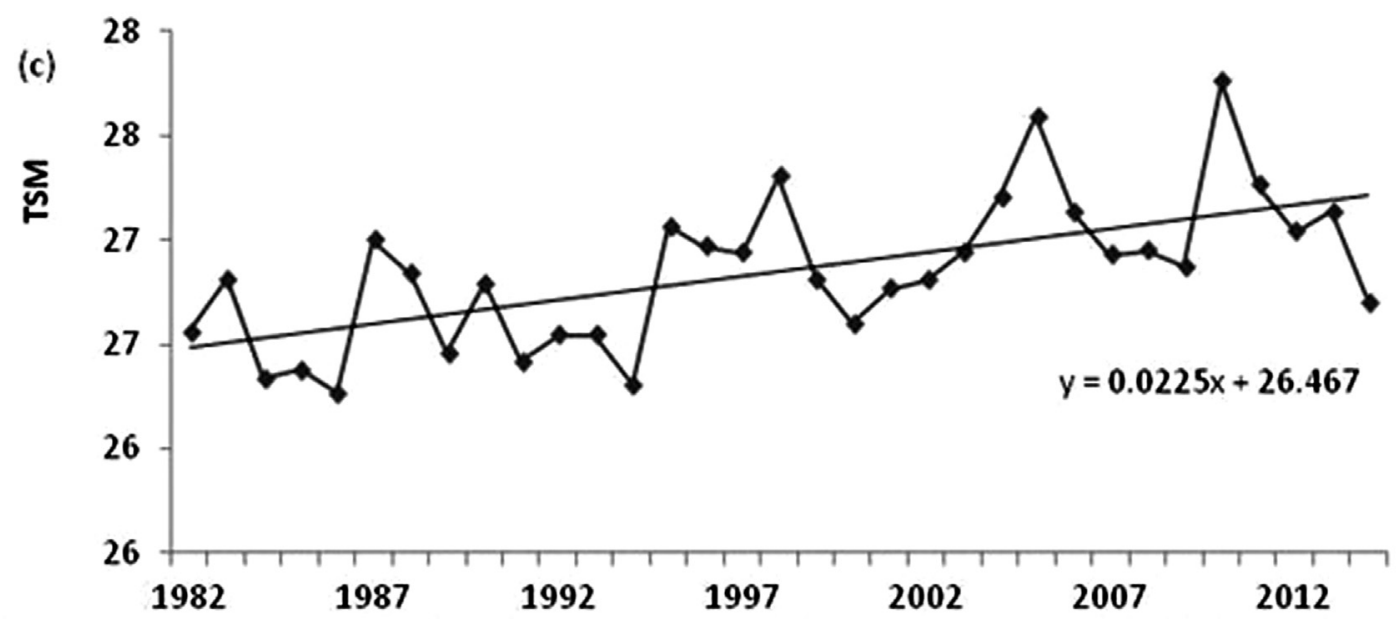

Figura 7 - Comportamento da TSM média anual na área norte (Dipolo) do Atlântico durante os anos de 1982 a 2014, juntamente com a tendência linear. 


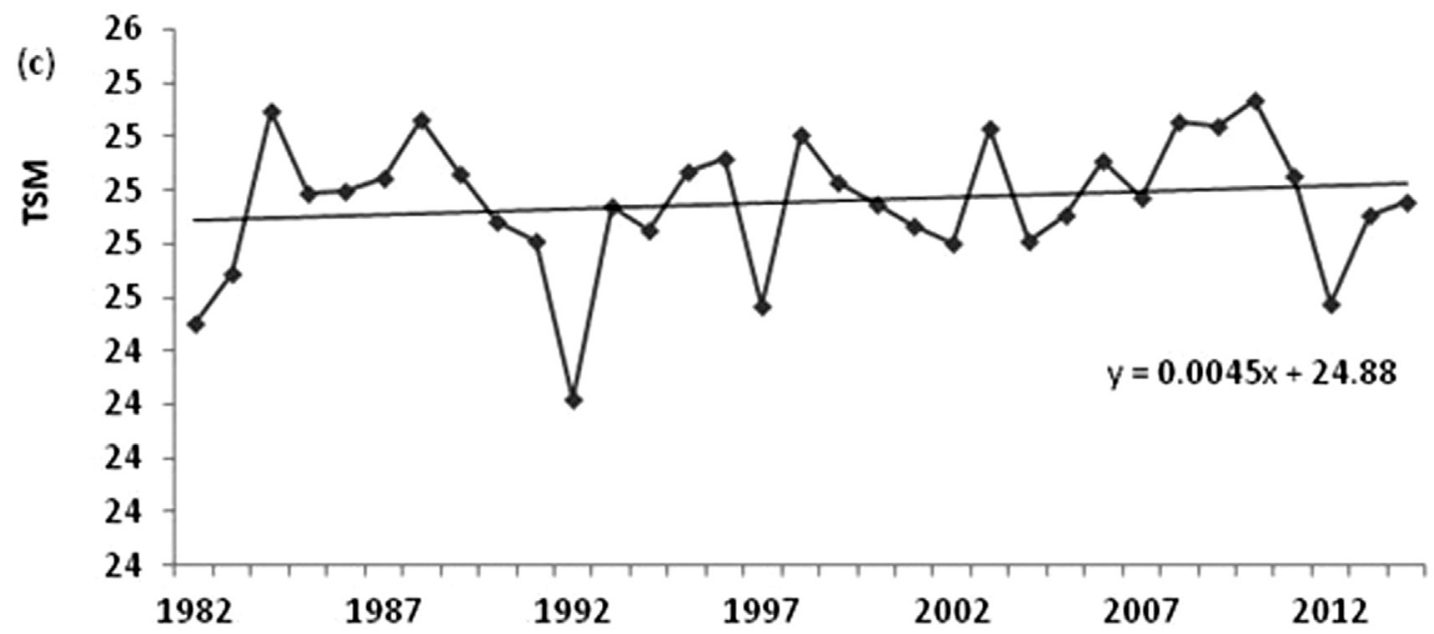

Figura 8 - Comportamento da TSM média anual na área sul (Dipolo) do Atlântico durante os anos de 1982 a 2014, juntamente com a tendência linear.

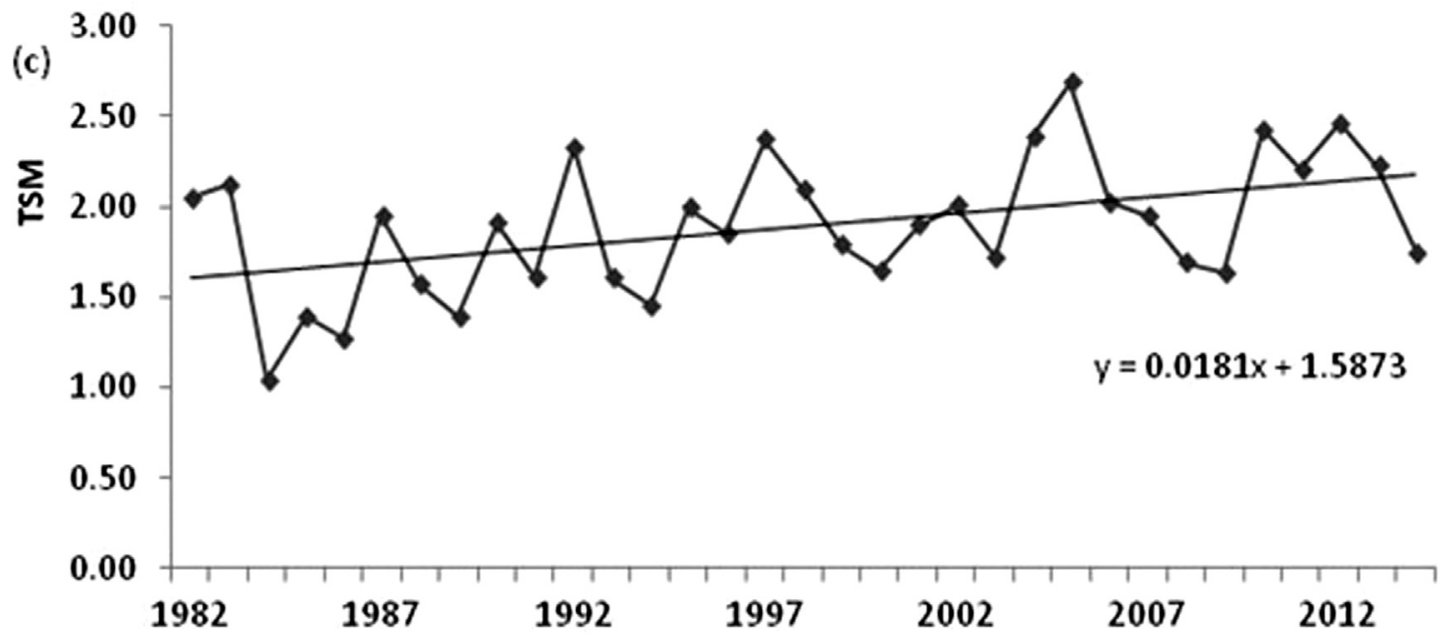

Figura 9 - Comportamento da TSM média anual na área do Dipolo (N-S) durante os anos de 1982 a 2014, juntamente com a tendência linear.

$1 \%$ pelo teste de Mann-Kendall. O ano de $1998\left(28,2{ }^{\circ} \mathrm{C}\right)$ foi o qual se observou o maior valor de TSM média anual, ou seja, o ano mais quente. Já o ano de $1985\left(27,4{ }^{\circ} \mathrm{C}\right)$ foi considerado com a menor TSM média anual, ou seja, o ano mais frio.

A década mais fria foi a de 80 , com uma média de $27,7{ }^{\circ} \mathrm{C}$ e a década de 2000 foi a mais quente, com uma média de $27,9^{\circ} \mathrm{C}$. As décadas 80 e 90 mostraram tendências lineares positivas. Por outro lado, a década de 2000 e entre os anos de 2010 a 2014, foram encontradas tendências lineares negativas.

\section{Conclusões e Recomendações}

Neste estudo foram avaliados as TSMs nos oceanos Pacífico e Atlântico Tropical, no período de 1982 a 2014,com o objetivo de se diagnosticar a tendência linear desta variável no período. Para isto usou-seo teste nãoparamétrico de Mann Kendall.
De acordo com os resultados obtidos, apenas a área norte (Dipolo) e o Dipolo (N-S) apresentaram tendências estatisticamente significativa ao nível de $1 \%$. Sendo que nestas duas áreas se observaram uma tendência linear positiva. Neste caso, visando validar os resultados da literatura citada, sugere-se um estudo futuro que investigue se esta tendência positiva de aumento da TSM em áreas do Oceano Atlântico Tropical implicou em tendência de redução das chuvas ocorridas na RSANEB neste período.

Com a aplicação da técnica dos quantis foi possível classificar os DN da média da TSM acumulada (de janeiro a junho) em anos quentes, normais e frios, bem como atestar a variabilidade de anos quentes ou frios para cada área. Assim, observou-se que os anos mais quentes, ou seja, com os maiores valores de DN de TSM no oceano Pacífico, foram 1987 e 1997, e no oceano Atlântico Tropical foi o ano de 2010. Os anos mais frios, ou seja, com menores valores de DN de TSM, no oceano Pacífico foram 1988, 1999 e 2007, e no oceano Atlântico foram os anos de 1986 e 
1992. Essa tendência positiva no Oceano Atlântico, em particular na sua área norte da bacia, é consistente com recentes estudos, que inferem essa mudança como possível efeito antropogênico [Servain et al. (2014)].

\section{Agradecimentos}

$\mathrm{O}$ autor principal agradece o apoio financeiro da Coordenação de Aperfeiçoamento de Pessoal de Nível Superior (CAPES) e a Agência Executiva de Gestão das Águas do Estado da Paraíba (AESA) pelo apoio logístico. Como também aos revisores do artigo pelas dicas.

\section{Referências}

AZIS, O.I.A.; BURN, D.H. Trends and variability in the hydrological regime of the Mackenzie River Basin. Journal of Hydrology, v.319, p.282-294, 2006.

CAVALCANTI, I.F.A.; MARENGO, J.A.; SATYAMURTY, P.; NOBRE, C.A.; TROSNIKOV, I.; BONATTI, J.P.; MANZI, A.O.; TARASOVA, T.; PEZZI, L.P.; ALMEIDA, C.; SAMPAIO, D.G.; CASTRO, C.C.; SANCHES, M.B.; CAMARGO, H. Global climatological features in a simulation using the CPTEC-COLA AGCM. Journal of Climate, 15(21), 2965-2988, 2002.

COELHO, C.A.S. Forecast calibration and combination: Bayesian assimilation of seasonal climate predictions. Ph.D. Thesis, Reading University, 2005.

COELHO C.A.S.; STEPHENSON, D. B.; DOBLAS-REYES, F. J.; BALMASEDA, M. From multi-model ensemble predictions to well-calibrated probability forecasts: Seasonal rainfall forecasts over South America 1959-2001. CLIVAR Exchanges No.32. v. 10, No. 1, 14-20, 2005.

COELHO C.A.S.; STEPHENSON, D.B.; BALMASEDA, M.; DOBLAS-REYES, F.J.; VAN OLDENBORGH, G.J. Toward an integrated seasonal forecasting system for South America. Journal of Climate, v. 19, p. 3704-3721, 2006.

ENFIELD, D.B.; MAYERr, D.A .Tropical Atlantic TSM variability and its relation to El Niño-Southern Oscillation. Journal of Geophysical Research Letter, v.102, p.929-945, 1997.

FOLLAND, C.K.; COLMAN, A.W.; ROWELL, D.P.; DAVEY, M.K. Predictability of northeast Brazil rainfall and real-time forecast skill, 1987-98. Journal of Climate, 14(9), p. 19371958, 2001.

GADGIL, A.; DHORDE, A.Temperature trends in twentieth century at Pune, India.Atmospheric Environment, v.39, n.35, p.6550 - 6556, 2005.

HARZALLAH, A.; ARAGÃO, J.O.R.; SADORNY, R. Interannual rainfall variability in northeast Brazil: Observation and model simulation. International Journal of Climatology, v.16, p.861-878, 1996.

HASTENRATH, S.; HELLER, L. Dynamics of climatic hazards in northeast Brazil.Quarterly Journal of the Royal Meteorological Society, v.103, p.77-92. 1977.
HASTENRATH, S. Prediction of Northeast Brazil rainfall anomalies.Journal of Climate, Boston, v.3, p. 893-904, 1990.

HASSEL, D.C.; WOODAGE, M.J. A high resolution atmospheric GCM for the generation of regional climate scenarios. Submitted to Climate Dynamics. Amsterdan, 2007.

KEENDALL, M.G. Rank Correlation Methods. London: Charles Griffin, 1975, 120p.

MANN, H.B.Econometrica. The econometric society, v.13, n.3, p. 245-259, 1945 .

MANTUA, N.J. et al. A Pacific interdecadal climate oscillation with impacts on salmon production. Bull. Amer. Meteor. Soc., Boston, v.78, p.1069-1079. 1997.

MARENGO, J.; CAVALCANTI, I.F.A.; SATYAMURTY, P.; TROSNIKOV, I.; NOBRE, C.A.; BONATTI, J.P.; CAMARGO, H.; SAMPAIO, G.; SANCHES, M.B.; MANZI, A.; CASTRO, C.A.C.; ALMEIDA, C.D.; PEZZI, L.P.; CANDIDO, L. Assessment of regional seasonal rainfall predictability using CPTEC/COLA atmospheric GCM. Climate Dynamics, 21(5-6), p. 459-475, 2003.

MODARRES R.; SILVA, V.P.R. Rainfall trends in arid and semi-arid regions of Iran. Journal of Arid Environments, v. 70, p.344-355, 2007.

MOURA, A.D.; HASTENRATH, S. Climate prediction for Brazil's Nordeste: Performance of empirical and numerical modeling methods. Journal of Climate, 17(13), p. 2667-2672, 2004.

MOURA, A.D., SHUKLA, J. On the dynamics of droughts in northeats Brazil: Observation, theory, and numerical experiments with a general circulation model. Journal of Atmospheric Sciences, v. 38, p.2653-2675, 1981.

NOBRE, C.A. Mudanças climáticas globais: possíveis impactos nos ecossistemas do País.

Parcerias Estratégicas, Brasília, n.12, p.239-58, 2001.

NOBRE, P.; SHUKLA, J. Variations of sea surface temperatures, wind stress, and rainfall over the tropical over the tropical Atlantic and South America. J. Climate, Boston, v.9, n.10, p.2464-2479, 1996.

SERVAIN, J. Simple climatic indices for the tropical Atlantic ocean and the some applications. Journal Geophyscal Research, v.96, n.8, p.15137-15146, 1993.

SERVAIN, J.; CANIAUX, G.; KOAUDIO, Y. V.; MAcPHADEN, M.; ARAÚJO, M. Recent climatic trend in the tropical Atlantic. Climate Dynamics, DOI 10.1007/s00382-014-2168-7. 2014.

SILVA, V.P.R. On climate variability in Northeast of Brazil.Journal of Arid Environments, v.58, n.4, p.575 - 596, 2004.

ZHANG, Y.; WALLACE, J.M.; BATTISTI, D.S. ENSO-like interdecadal variability. J. Climate, Boston, v.10, p.1004-1020. 1997.

YUE, S.; PILON P.; CAVADIAS, G.Corrigendum to "Power of the Mann-Kendall and Spearman's rho tests for detecting monotonic trends in hydrological series". Journal of Hydrology, v.264, n.1 - 4, p.262-263, 2002.

All the contents of this journal, except where otherwise noted, is licensed under a Creative Commons Attribution License CC-BY. 